\title{
Assessment of Electromagnetic Absorption towards Human Head Using Specific Absorption Rate
}

\author{
Abdul Rashid O. Mumin, R. Alias, Jiwa Abdullah, Samsul Haimi Dahlan, Jawad Ali \\ Wireless Radio Science Centre(WARS), Research Centre for Applied Electromagnetics, Faculty of Electrical and \\ Electronic Engineering, Universiti Tun Hussein Onn Malaysia, \\ Johor, Malaysia
}

\begin{tabular}{l} 
Article Info \\
\hline Article history: \\
Received Aug 21, 2018 \\
Revised Oct 26, 2018 \\
Accepted Nov 14, 2018 \\
\hline
\end{tabular}

\section{Keywords:}

ISM

Multilayer head phantom

Specific Absorption Rate

Square ring antenna

\begin{abstract}
This paper presents a compact square slot patch antenna characterstics for wireless body area network (WBANs) applications. The assessment of the effects of electromagnetic energy (EM) on the human head is necessary because the sensitivity of human head to high radiation level. Although, structuring of low EM antennas is a major problem in the improvement of portable device and reducing the size of of the antenna is a major concern. However, performance of antenna reduces when antenna operates near human body which is lossy and complex in nature. The proposed antenna operates at $5.8 \mathrm{GHz}$ of the ISM Band for WBAN applications. The antenna has been designed and simulated with two different types of multilayer human head phantoms to characterize the antenna near the human head.The multilayer head phantom is constructed by five layers tissues head model using CST Microwave studio. Therefore, antenna with spherical phantom has the highest SAR value $0.206 \mathrm{~W} / \mathrm{Kg}$, while antenna with cubical phantom contributed the lowest SAR value of 0.166 for $10 \mathrm{~g}$ tissue at $5.8 \mathrm{GHz}$ frequency exposed, whereas, the antenna with cubical phantom and spherical phantom have gain of $6.46 \mathrm{dBi}$ and $6.2 \mathrm{dBi} \mathrm{GHz}$ respectively. It was observed that antenna performance significantly increased. The presented prototype has a potential to work for ISM applications.
\end{abstract}

Copyright $\odot 2018$ Institute of Advanced Engineering and Science. All rights reserved.

\section{Corresponding Author:}

Abdul Rashid O. Mumin,

Wireless Radio Science Centre(WARS), Research Centre for Applied Electromagnetics,

Faculty of Electrical and Electronic Engineering,

Universiti Tun Hussein Onn Malaysia, Johor, Malaysia

Email: Abdulrashidomar3@gmail.com

\section{INTRODUCTION}

The possible health risk of radio frequency (RF) wave radiated from antenna to the human body has appeared as a general concern. The growing international movement in wireless body area networks (WBANs), wireless personal area networks (WPANs) has increased interest in usable antenna designed for human body [1]. The future Improvement portable wireless will play an important role in the field of wireless communication prosperity and security, indoor constraint and arranging, welfare and social protection, for instance, biomonitoring [2-4]. The fundamental challenge in optimizing a portable antenna is the reduction of the negative effects of electromagnetic interaction (EM) between the proposed antenna and biological tissue [5].To efficiently explore a usable and built-in antenna for a body-centered wireless communication system, it is significant to assess the interaction between the human body and the electromagnetic (EM) wave emitted by the antenna. Interaction represents two different types: The effect of a human head on the performance of the antenna, and the effect of EM waves on the human body. In order to investigate the performance of antenna and the effect of EM, it must utilize a human head phantom as validation tool before the antenna is applied to the human body. From the literature, it has been observed that most of the researchers considered 
microstrip patch antenna as one of the most suitable candidates for portable applications due to low profile, easy to integrate and cost-effectiveness [6].

Several antennas have been studied; including EBG configuration for SAR reduction being presented [7]. RF simulation is performed for an eight-element SIR Head coil combined with a CP patch antenna for MRI at $298 \mathrm{MHz}$ [8]. UWB antenna for BAN application based on the EM radiation and SAR limits is proposed [9]. A two-turn external loop antenna with and without the magnetic core, which transfers more power compared to the simple loop antenna, due to the low and uniform SAR distribution is presented [10]. To understand the effects of the antenna position on the phone, the SAR result is compared even when the phone is reversed [11]. A fractal-based dual-band antenna integrated with a square sized EBG for portable applications [12]. The entire body calculates the average specific absorption rate estimated using a distributed personally distributed exposimeter [13].

Numerous wearable antennas have been suggested to BAN application in the form of monopole antenna [16], and a configuration of inverted-F antenna designs have been proposed [17]. However, they were neither unremarkable nor compact. Microstrip monopoles planar has been proposed [18], [19], and inverted-F antennas which have remarkable, but they have low efficiency due to radiation of important total of energy into the human body. Hence, they need to be positioned some separation far from the human body.

This paper presents the impact of electromagnetic radiation on the human head and it's on/off body performance in detuning of the antenna resonant frequency point.The comparison on/off- performance between in free space and the presence of human head are investigated and the antenna is operating at 5.8 $\mathrm{GHz}$ of the ISM (5.725-5.85 GHz). Therefore, two different types of multilayer head phantoms such as cubical head phantom and the spherical head phantom model are used for evaluating the SAR value in the numerical modeling. The proposed antenna is simulated with five significant layers of human head which includes skin, fat, bone, cerebrospinal fluid (CSF) and brain have been developed. The structures are designed and analyzed using CST microwave software and MATLAB.The paper is structured as follows: Antenna configuration and proposed human head Phantom in section 2.In section 3 results and analysis of the simulation of the antenna is performed, the comparison of the simulated and measured result of the antenna is presented, while the reduction of Specific Absorption Rate (SAR) in the presence of human head is discussed in section. At long last, in section 4 summaries of the outcome and findings will be concluded with the given future directions.

\section{ANTENNA CONFIGURATION AND PROPOSED HUMAN HEAD PHANTOM}

The physical shape of the antenna configuration is designed and optimized using CST microwave studio as shown in Figure 1.The demonstrated antenna is designed to operate at $5.8 \mathrm{GHz}$ for ISM band. FR4 substrate is chosen as the substrate, which has thickness of $1.6 \mathrm{~mm}$, a relative permitivity of 4.3 , and loss tangent of 0.025 . The proposed antenna has a minimized size of $11.45 \times 11.45 \mathrm{~mm}^{2}$. The designed antenna consists of a square patch with slot of $(\mathrm{Wr}=1.60 \mathrm{~mm})$ is embedded at the center of the patch. The simulated values of antenna parameters are shown in Table 1.

Table 1. Simulated values of Antenna Parameters

\begin{tabular}{ccc}
\hline Parameters & Symbol & Value unit $\left(\mathrm{mm}^{2}\right)$ \\
\hline Substrate & L1 xW1 & $11.45 \times 11.45$ \\
Feed Length & Lf & 9.43 \\
Height & $\mathrm{h}$ & 1.6 \\
Permittivity & $\mathrm{Er}$ & 4.3 \\
Tan $\delta$ & $\delta$ & 0.0025 \\
Ring slot & $\mathrm{Wr}$ & $1.60 \times 1.60$ \\
Feedline width & $\mathrm{Wf}$ & 1.40 \\
\hline
\end{tabular}

BEEI, Vol. 7, No. 4, December 2018: 657 - 664 


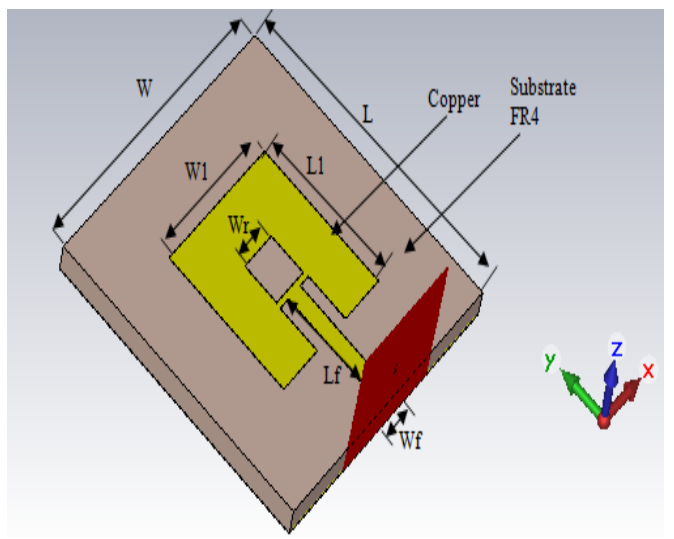

Figure 1. Top view of printed antenna

The proposed antenna has the dimensions L1xW1, where L1 is in charge of the current resonant frequency and the width of the patch has small impact. To design antenna parameters on the design frequency $5.8 \mathrm{GHz}$ can be used by the following equations [20]:

$$
\begin{aligned}
& \mathrm{W} 1=\frac{\mathrm{C}}{2 \mathrm{fr}} \sqrt{\frac{2}{\varepsilon_{r}+1}} \\
& L_{1}=L_{\text {eff }}-2 \Delta l
\end{aligned}
$$

where $L_{l}$ is the current length of square patch, $W_{l}$ is the specific width of the square patch, $\varepsilon_{\text {eff }}$ is the actual effective dielectric constant while $L_{\text {eff }}$ and $\Delta l$ are the specific length extension and actual of effective length of the patch.

\subsection{Specific Absorption Rate (SAR) and Proposed Phantom Model of Human Head}

The specific absorption rate (SAR) is the main parameter for characterizing the power consumed by human head and bodies under electromagnetic radiation. It is understood that the complex configuration of the antenna with the modeling of human head indicates a very complex SAR evaluation. Thus, the numerical modeling requires a high source of computational and a long simulation in numerical modeling. Despite the fact that the SAR is determined by the RF energy level caused by any part of the body, its values are typically measured for $1 \mathrm{~g}$ or $10 \mathrm{~g}$ of cube tissue simulation and the SAR limit set by the FCC and the European Uninon is $1.6 \mathrm{~W} / \mathrm{kg}$ and $2 \mathrm{~W} / \mathrm{kg}$ averaged over 1 gram averaged over $10 \mathrm{~g}$ of actual tissue respectively [21].

$$
S A R=\frac{\sigma \mathrm{E}^{2}}{\rho}
$$

where $\rho, \sigma$ and $\mathrm{E}$ are body tissue density in $\mathrm{Kg} / \mathrm{m}^{3}$, electrical conductivity of body tissues in $\mathrm{S} / \mathrm{m}$ and square root of electrical tissue V/m rms. The IEEE C 95.3 standard used for all simulations as the average method for calculating SAR values with radiated power $0.1 \mathrm{~W}$.

There are several approaches to SAR evaluation; In general, numerical modeling and experimental measurements are two major trends in SAR analysis. Configurations of the phantom heads, such as cubical and spherical human head models, are often used to evaluate SAR values in numerical modeling.For various forms of the configuration of human head model, their far-field radiation pattern is also expected to be different due to the scattering and diffraction at the interface between the phantom and the free space beyond. Two types of configuration of human head models presented in this study, namely the cubical human head phantom and spherical human head phantom model; the cubical phantom with dimensions $47 \mathrm{~mm} \times 47 \mathrm{~mm} \times$ $23.50 \mathrm{~mm}^{3}$, the spherical phantom with a radius of $47 \mathrm{~mm}$ is based on the shape of a typical adult human head. Therefore, these phantoms are used for evaluating SAR values as shown in Figure 2. Table 2 shown dielectric properties of biological tissue used in the head model at $5.8 \mathrm{GHz}$ with $100 \mathrm{~mW}$ power [19]. 


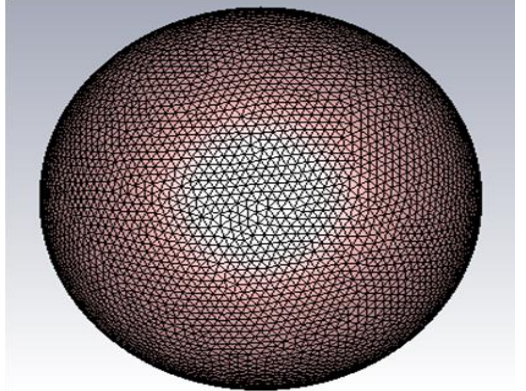

(a)

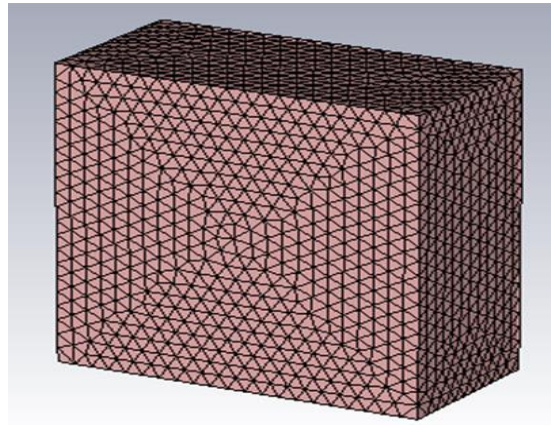

(b)

Figure 2. Proposed phantom models of human head (a) spherical phantom (b) cubical phantom

Table 2. Dielectric Properties of Biological Tissue Used in the Head Model at $5.8 \mathrm{GHz}$ with 100mW

\begin{tabular}{cccc}
\multicolumn{4}{c}{ Power [19] } \\
\hline Tissue & Relative Permittivity $(\varepsilon r)$ & Tan $\delta$ & Thickness $\left(\mathrm{mm}^{2}\right)$ \\
\hline Skin & 35.11 & 0.33 & 2 \\
Fat & 9.86 & 0.26 & 2 \\
Bone & 9.67 & 0.37 & 7 \\
CSF & 60.47 & 0.40 & 1 \\
Brain & 44.00 & 0.35 & 35 \\
\hline
\end{tabular}

\section{RESULTS AND ANALYSIS}

This section clarifies the results of outcome and it the meantime is given at the complete discussion. Results are represented in figures, graphs. The discussion can be made a few sub-topics.

\subsection{Simulated and Measured Results of the Antenna in Free Space Characterization}

This section analyses the square ring antenna performance and compares with the simulation and measurement results obtained by using CST based on finite different time domain and variety of antenna parameters are illustrated by using MATLAB. The simulated and measured return loss characteristics of presented antenna printed on FR4 substrate in the free space are shown in Figure 3 (a). Simulation and measurement return loss show that the result covers the entire ISM band $(5.725-5.875) \mathrm{GHz}$. Thus, the simulated return loss is about $-42.821 \mathrm{~dB}$ while the measured return loss is about $-20.46 \mathrm{~dB}$ and the bandwidths of $263.3 \mathrm{MHz}$ and $280 \mathrm{MHz}$ respectively. The measured result for S11 is moved slightly towards the right side of $5.8 \mathrm{GHz}$ frequency due to farbrication tolerance and dielectric loss of material. Therefore, simulated and measured VSWR of presented antennas are lower than 2 as shown in Figure 3 (b).

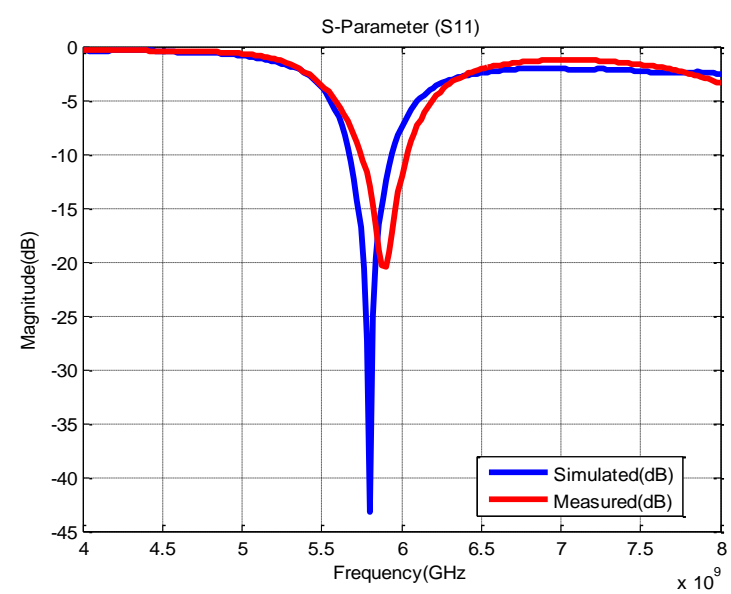

(a)

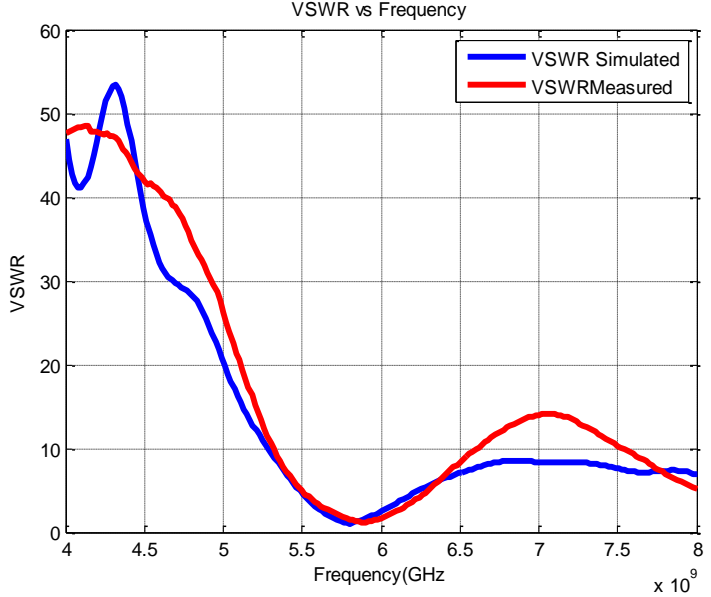

(b)

Figure 3. Comparison simulated and measured results of proposed antenna in free space (a) S11 and (b) VSWR 


\subsection{Performance of Antenna on Human Head Phantom and SAR Analysis}

The performance of antenna on human head as a system worn on the body will lead to a various of potential issues. When the presented antenna is designed and mounted on the human head, frequency detuning happens due to lossy characterstics of the human head. Two different types of multilayer human head phantoms are utilized to characterize the antenna near the human head, in order to decrease the complexity of the compution process. These phantoms includes skin, fat, bone, Cerebrospinal fluid (CSF) and brain layers are modeled as shown in Figure 2. The instance of $5.8 \mathrm{GHz}$ antenna, the magnitudes of the $S_{11}$ are below $-10 \mathrm{~dB}$ for the patch antenna with the nearness of two different types of human head phantoms as shown in Figure 4.In general, results have demonstrated that the $S_{11}$ magnitude of both cubical and spherical phantoms are different because of the nearness phantom head models with high permittivity characterstics. As shown in Figure 4, results have shown that the resonant frequencies of the orginal patch antenna at 5.8 $\mathrm{GHz}$ with the magnitude of return loss $-42.821 \mathrm{~dB}$ while the cubical and spherical phantom with the magnitude of resturn loss $-28.86 \mathrm{~dB}$ and $-30.67 \mathrm{~dB}$ respectively.

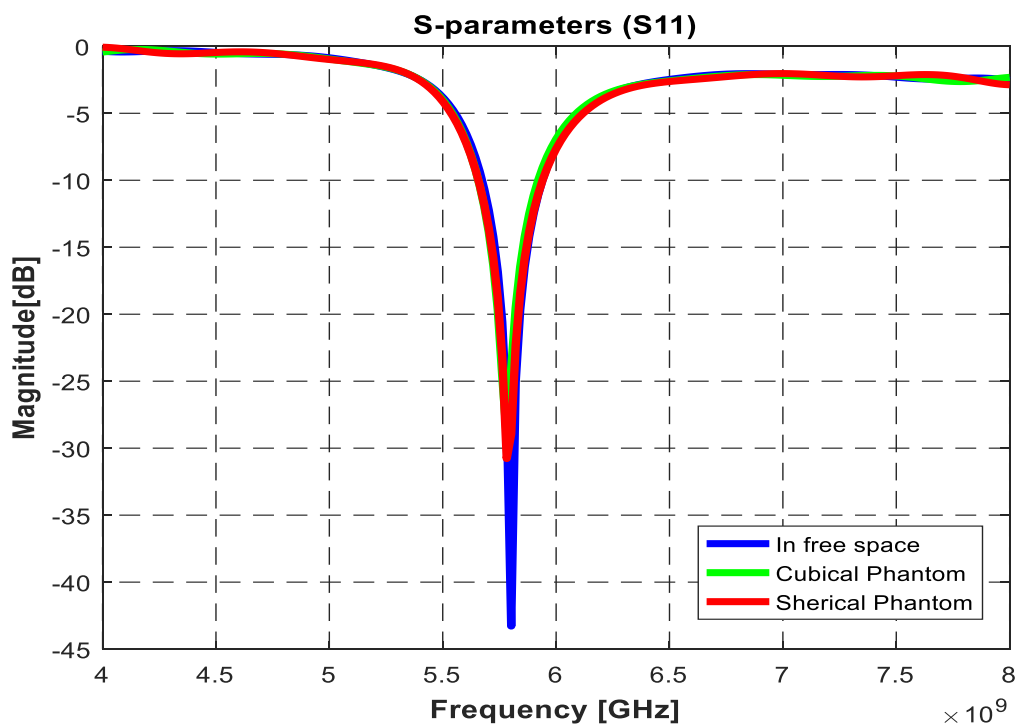

Figure 4. Comparison of the simulated reflection coefficient of the designed square ring antenna in free spaces and human head

Comparison of the maximum average SAR over $10 \mathrm{~g}$ of tissue induced in two different human head phantoms in the near-field of $5.8 \mathrm{GHz}$ antenna has investigated by using Finite Difference Time-domain (FDTD) method.The multilayered phantom consists of different tissues which were utilized to determine the peak SAR values averaged over 10-g and all of each tissue is based on their electromagnetic properties and set at $5.8 \mathrm{GHz}$. The maximum absorption rate (SAR) is simulated by considering the average limits of $2 \mathrm{~W} / \mathrm{kg}$ of the ICNIRP on 10 gram of tissue. The designed antenna should comply with the SAR value given by The IEEE C95.3 standard utilized for all simulations, as the average method for calculating SAR values with transmitted power $0.1 \mathrm{~W}$. The result has shown that the SAR value is varied for different types of phantom head models due to the shapes and geometry of the phantom. The skin as the highest SAR occurs on its surface in real practice when the antenna is closed in proximity to the head model and the separation between head and antenna is $0 \mathrm{~mm}$. It is also observed that the SAR value induced in two types of multilayer head phantom is almost reduced when it is used as EPDM foam as isolating layer. The main reason for using the foam is to prevent direct contact of the antenna with the presence of the human head as isolating material. The dielectric properties of $\varepsilon r=1.3$ and Tan $\delta=0.0015$ for EPDM at $5.8 \mathrm{GHz}$. It can be seen that SAR over $10 \mathrm{~g}$ tissue induced in all $0.206 \mathrm{~W} / \mathrm{kg}, 0.166 \mathrm{~W} / \mathrm{kg}$ an in spherical phantom and cubical phantom respectively. Figure 5 indicates the simulated SAR for 10-g tissue when antenna is mounted on the human head. 


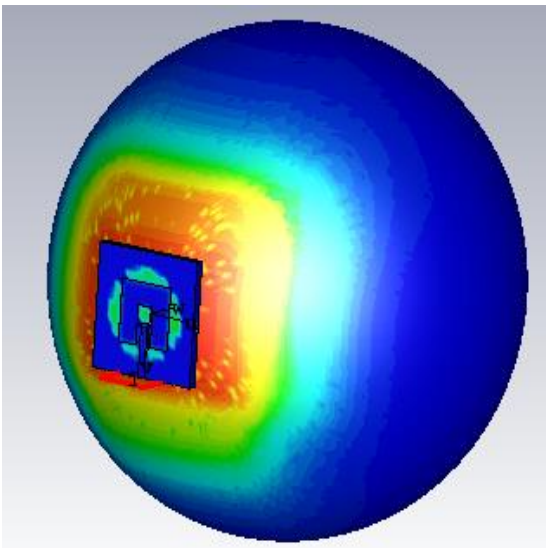

(a)

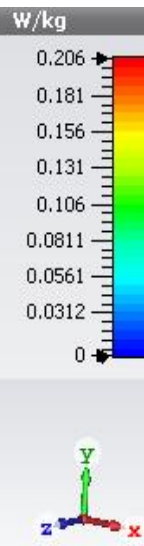

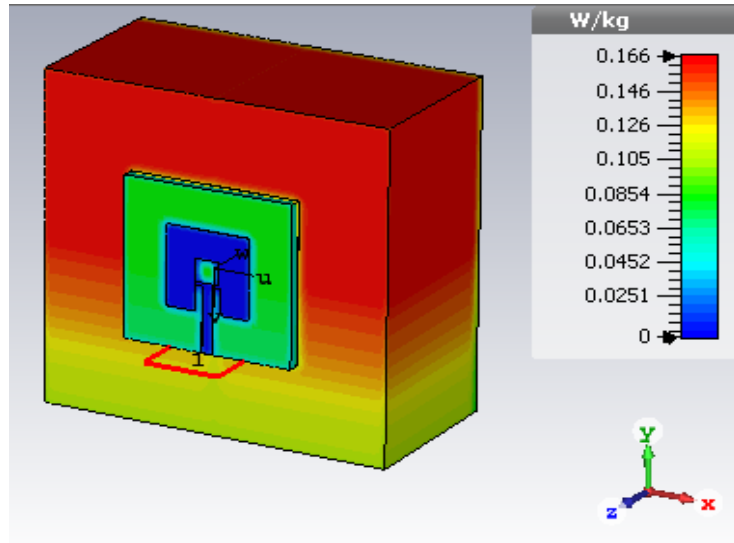

(b)

Figure 5. Comparison simulated SAR value of antenna mounted on human head (a) spherical phantom and

(b) cubical phantom

Figure 6 indicates the simulated SAR distribution at $5.8 \mathrm{GHz}$ against separation distance between head and antenna.It can be recognized that the antenna with cubical phantom produced the peak SAR values compared with spherical phantom at $5.8 \mathrm{GHz}$ for $10 \mathrm{~g}$ tissue.

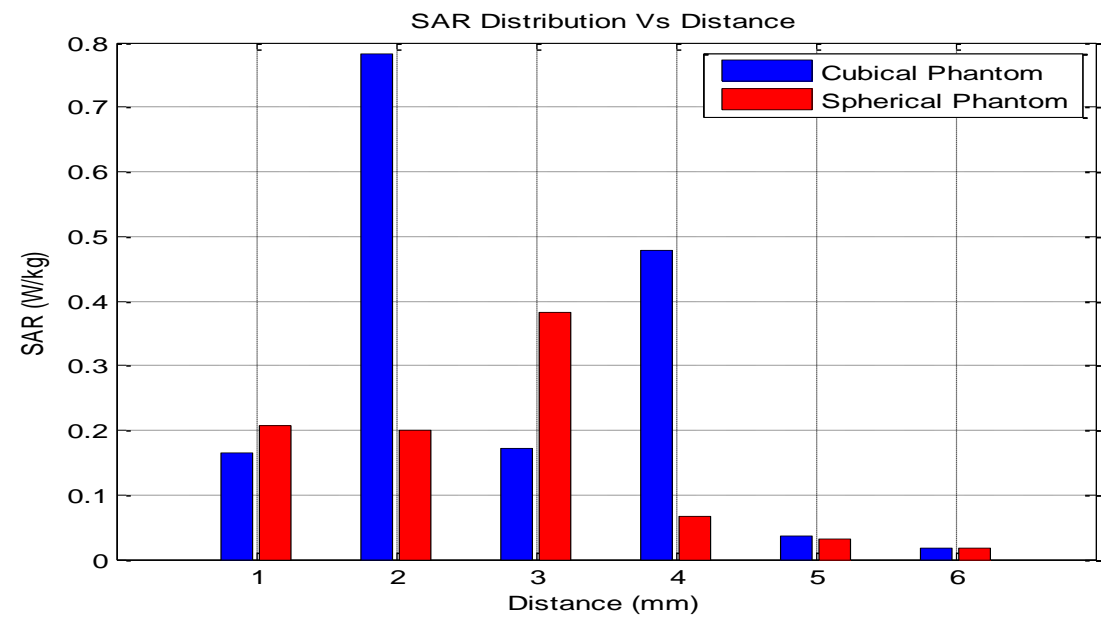

Figure 6. Comparison of SAR variation against distance between human head and antenna

The radiation characteristics of the presented antenna is effected due to presence of various human head phantoms, the results indicated that radation characteristics changes take place due to various shapes of human head as the square ring antenna is placed on the human head and that various positions result in the direction of the main beam. The cubical and spherical human head phantom have the greatest effect on the far field radiation pattern in general,the result of radiation pattern reveals that the gain of the antenna with mounted cubical human head phantom is increased from $5.96 \mathrm{dBi}$ to $6.46 \mathrm{dBi}$ compared in free space as illustrated as shown in Figure 7. 


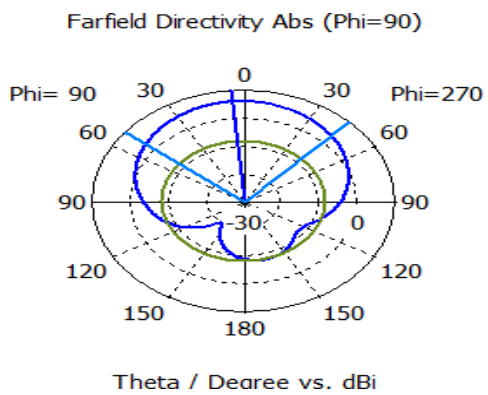

(a)

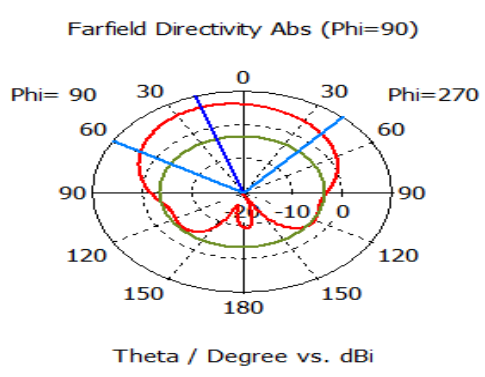

(b)

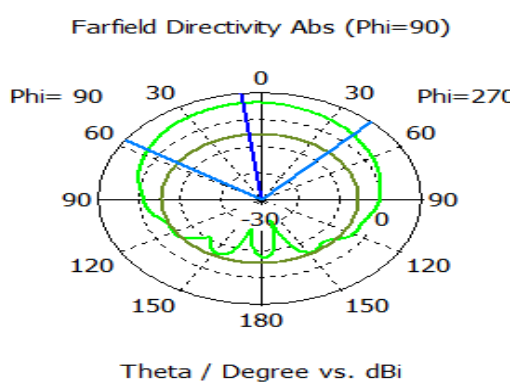

(c)

Figure 7. Comparison of Simulated Radiation characteristics of the proposed antenna (a) in free space (b) Cubical phantom and (C) Spherical Phantom at 5.8 GHz

\section{CONCLUSION}

A low profile antenna useful in wireless body area network (WBAN) has presented in this paper. The effect of electromagnetic (EM) radiations emit from antenna on human head has been investigated. To analyze the specific absorption rate (SAR) simulation, a square patch antenna is presented with pros, especially in ISM band applications. Although antenna size reduction is a distinguish topic of antenna development, the antenna performance significantly drops for a small antenna and the proposed antenna resonates at $5.8 \mathrm{GHz}$ of the ISM band for WBAN applications. Two types of the multilayered human head phantoms are used, with an aim to determine the maximum SAR value for $2 \mathrm{~W} / \mathrm{kg}$ averaged over $10 \mathrm{~g}$ of tissue with $100 \mathrm{~mW}$ antenna power. It has been recognized that some pattern changes occur as the square ring is placed on the human head. Hence, EPDM foam isolator layer is used as isolating material and this layer reduced the SAR value. Thus, spherical phantom has higher SAR value $0.206 \mathrm{~W} / \mathrm{Kg}$, while cubical phantom contributed the lower SAR value of 0.166 for $10 \mathrm{~g}$ tissue at $5.8 \mathrm{GHz}$ frequency exposed. The cubical phantom and spherical phantom have gain of $6.46 \mathrm{dBi}$ and $6.2 \mathrm{dBi} \mathrm{GHz}$ respectively. The proposed antenna has been designed using CST and variation of parameters of antenna is computed using MATLAB, while the physical antenna layout is fabricated on FR4 substrate having a miniaturized size of $11.45 \times 11.45 \mathrm{~mm}^{2}$. A good agreement between simulation and measurements was obtained. The presented prototype has a potential to work for ISM applications.

\section{ACKNOWLEDGEMENT}

The authors would like to thank (ORICC) UTHM fellowship Grant Vote U585. This paper was partly sponsored by Research Management Centre (RMC) UTHM.

\section{REFERENCES}

[1] Zheng, Ya-Li, Xiao-Rong Ding, Carmen Chung Yan Poon, Benny Ping Lai Lo, Heye Zhang, Xiao-Lin Zhou, Guang-Zhong Yang, Ni Zhao, and Yuan-Ting Zhang. Unobtrusive sensing and wearable devices for health informatics." IEEE Transactions on Biomedical Engineering 2014; 61 (5): 1538-1554.

[2] Movassaghi, Samaneh, Mehran Abolhasan, Justin Lipman, David Smith, and Abbas Jamalipour. "Wireless body area networks: A survey." IEEE Communications Surveys \& Tutorials. 2014; 16 (3): 1658-1686.

[3] Yoo, Hoi-Jun. "Your heart on your sleeve: advances in textile-based electronics are weaving computers right into the clothes we wear." IEEE Solid-State Circuits Magazine. 2013; 5(1): 59-70.

[4] Januszkiewicz, Łukasz, Paolo Di Barba, and Sławomir Hausman. "Field-based optimal placement of antennas for body-worn wireless sensors." Sensors. 2016;16( 5): 713.

[5] Koski, K., L. Sydänheimo, Y. Rahmat-Samii, and L. Ukkonen. "Fundamental characteristics of electro-textiles in wearable UHF RFID patch antennas for body-centric sensing systems." IEEE Transactions on Antennas and Propagation. 2014; 62(12): 2014: 6454-6462.

[6] Raad, Haider R., Ayman I. Abbosh, Hussain M. Al-Rizzo, and Daniel G. Rucker. "Flexible and compact AMC based antenna for telemedicine applications." IEEE Transactions on antennas and propagation. 2013; 61(2): 524531.

[7] Yan, Sen, Ping Jack Soh, and Guy AE Vandenbosch. "Low-profile dual-band textile antenna with artificial magnetic conductor plane." IEEE Transactions on Antennas and Propagation. 2014; 62(12): 6487-6490.

[8] Cook, Benjamin Stassen, and Atif Shamim. "Utilizing wideband AMC structures for high-gain inkjet-printed antennas on lossy paper substrate." IEEE Antennas and Wireless Propagation Letters. 2013:12: 76-79. 
[9] Saeed, Saud M., Constantine A. Balanis, and Craig R. Birtcher. "Inkjet-printed flexible reconfigurable antenna for conformal WLAN/WiMAX wireless devices." IEEE Antennas and Wireless Propagation Letters. 2016; 15: 19791982.

[10] $M$ Makinen, Riku M., and Tiiti Kellomaki. "Body effects on thin single-layer slot, self-complementary, and wire antennas." IEEE Transactions on Antennas and Propagation .2014;62(1): 385-392

[11] Velan, Sangeetha, Esther Florence Sundarsingh, Malathi Kanagasabai, Aswathy K. Sarma, Chinnambeti Raviteja, Ramprabhu Sivasamy, and Jayaram Kizhekke Pakkathillam. "Dual-band EBG integrated monopole antenna deploying fractal geometry for wearable applications." IEEE Antennas and Wireless Propagation Letters. 2015; 14: 249-252.

[12] Samal, Purna B., Ping Jack Soh, and Guy AE Vandenbosch. "UWB all-textile antenna with full ground plane for off-body WBAN communications." IEEE transactions on antennas and propagation. 2014;62(1): 102-108.

[13] Samal, Purna B., Ping Jack Soh, and Guy AE Vandenbosch. "UWB all-textile antenna with full ground plane for off-body WBAN communications." IEEE transactions on antennas and propagation. 2014;.62( 1): 102-108.

[14] El Hajj, W., C. Person, and J. Wiart. "A novel investigation of a broadband integrated inverted-F antenna design; application for wearable antenna." IEEE Transactions on Antennas and Propagation. 2014;62(7): 3843-3846.

[15] Soh, Ping Jack, Guy AE Vandenbosch, Soo Liam Ooi, and Nurul Husna Mohd Rais. "Design of a broadband alltextile slotted PIFA." IEEE Transactions on Antennas and Propagation. 2012; 60(1): 379-384.

[16] Agneessens, Sam, Sam Lemey, Thomas Vervust, and Hendrik Rogier. "Wearable, small, and robust: The circular quarter-mode textile antenna." IEEE Antennas and Wireless Propagation Letters. 2015;14: 1482-1485.

[17] Scarpello, Maria Lucia, Luigi Vallozzi, Hendrik Rogier, and Dries Vande Ginste. "High-gain textile antenna array system for off-body communication." International Journal of Antennas and Propagation (2012).

[18] Kang, Seonghun, and Chang Won Jung. "Wearable fabric reconfigurable beam-steering antenna for on/off-body communication system." International Journal of Antennas and Propagation (2015)

[19] Björninen, Toni, and Fan Yang. "Low-profile head-worn antenna with a monopole-like radiation pattern." IEEE antennas and wireless propagation letters. 2016;15: 794-797.

[20] Mumin, Abdul Rashid O., Rozlan Alias, Jiwa Abdullah, Samsul Haimi Dahlan, Raed Abdulkareem Abdulhasan, and Jawad Ali. "Simulation of Square Ring Microstrip Patch Antenna Performance Based on Effects of Various Dielectric Substrates." In Asian Simulation Conference, Singapore, Springer, 2017,.pp. 679-694.

[21] Sallomi, Adheed Hasan. "A theoretical approach for SAR calculation in human head exposed to RF signals." Journal of Engineering and Sustainable Development 2012; 16(4): 304-313. 